\title{
Intestinal parasitic infections in schoolchildren in different settings of Côte d'Ivoire: effect of diagnostic approach and implications for control
}

Jean T Coulibaly ${ }^{1,2,3,4^{*}}$, Thomas Fürst ${ }^{1,2}$, Kigbafori D Silué ${ }^{3,4}$, Stefanie Knopp ${ }^{1,2}$, Dimitri Hauri ${ }^{1,2}$, Mamadou Ouattara ${ }^{3}$, Jürg Utzinger ${ }^{1,2}$ and Eliézer K N'Goran ${ }^{3,4}$

\begin{abstract}
Background: Social-ecological systems govern parasitic infections in humans. Within the frame of assessing the accuracy of a rapid diagnostic test for Schistosoma mansoni in Côte d'Ivoire, three different endemicity settings had to be identified and schoolchildren's intestinal parasitic infection profiles were characterized.

Methods: In September 2010, a rapid screening was conducted in 11 schools in the Azaguié district, south Côte d'Ivoire. In each school, 25 children were examined for S. mansoni and S. haematobium. Based on predefined schistosome endemicity levels, three settings were selected, where schoolchildren aged 8-12 years were asked to provide three stool and three urine samples for an in-depth appraisal of parasitic infections. Triplicate Kato-Katz thick smears were prepared from each stool sample for S. mansoni and soil-transmitted helminth diagnosis, whereas urine samples were subjected to a filtration method for S. haematobium diagnosis. Additionally, a formol-ether concentration method was used on one stool sample for the diagnosis of helminths and intestinal protozoa. Multivariable logistic regression models were employed to analyse associations between schoolchildren's parasitic infections, age, sex and study setting.

Results: The prevalences of S. mansoni and S. haematobium infections in the initial screening ranged from nil to $88 \%$ and from nil to $56 \%$, respectively. The rapid screening in the three selected areas revealed prevalences of S. mansoni of $16 \%, 33 \%$ and $78 \%$. Based on a more rigorous diagnostic approach, the respective prevalences increased to 33\%, 53\% and 92\% S. haematobium prevalences were $0.8 \%, 4 \%$ and $65 \%$ (rapid screening results: $0.0 \%$, $0.0 \%$ and $54 \%$ ). Prevalence and intensity of Schistosoma spp., soil-transmitted helminths and intestinal protozoan infections showed setting-specific patterns. Infections with two or more species concurrently were most common in the rural setting (84\%), followed by the peri-urban (28\%) and urban setting (18\%).
\end{abstract}

Conclusions: More sensitive diagnostic tools or rigorous sampling approaches are needed to select endemicity settings with high fidelity. The observed small-scale heterogeneity of helminths and intestinal protozoan infections has important implications for control.

\section{Background}

Intestinal parasitic infections (e.g. helminths and pathogenic intestinal protozoa) are of considerable public health importance, particularly in developing countries. For example, the global burden caused by soiltransmitted helminthiasis (infections with Ascaris

\footnotetext{
* Correspondence: jean.coulibaly@unibas.ch

'Department of Epidemiology and Public Health, Swiss Tropical and Public Health Institute, P.O. Box, CH-4002 Basel, Switzerland

${ }^{2}$ University of Basel, P.O. Box, CH-4003 Basel, Switzerland

Full list of author information is available at the end of the article
}

lumbricoides, hookworm and Trichuris trichiura) is estimated at 39 million disability-adjusted life years (DALYs), whereas the burden due to schistosomiasis (mainly Schistosoma mansoni, S. haematobium and S. japonicum) is estimated at 4.5 million DALYs [1,2]. Amoebiasis due to infections with the intestinal protozoon Entamoeba histolytica results in 40,000100,000 deaths each year [3], and giardiasis due to Giardia intestinalis might affect 200 million people per annum [4]. However, the burden of pathogenic intestinal protozoan infections in terms of DALYs remains to be

\section{Biomed Central}


determined, which is a challenge due to the paucity of up-to-date epidemiological data [5].

Parasitic infections are governed by behavioural, biological, environmental, socioeconomic and health systems factors. Local conditions, including access to and quality of domestic and village infrastructure, economic factors such as disposable income, employment and occupation, and social factors such as education, influence the risk of infection, disease transmission and associated morbidity and mortality [6-8].

As a preparatory step for a rigorous assessment of the accuracy of a new rapid diagnostic test for S. mansoni in Côte d'Ivoire, we aimed to identify a low prevalence (10-24\%) and a moderate prevalence (25-49\%) setting for S. mansoni, and a third setting where S. mansoni and S. haematobium co-exist. While in the initial screening only single stool and urine samples were collected from each individual. In the main study, the diagnosis was based on multiple stool and urine examinations to more accurately characterize schoolchildren's intestinal parasitic infection profiles in the selected study areas. Implications of our findings for the design, implementation and monitoring of intestinal parasite control strategies in Côte d'Ivoire are discussed.

\section{Methods}

\section{Ethical considerations}

This study was approved by the institutional research commission of the Swiss Tropical and Public Health Institute (Basel, Switzerland) and received clearance from the ethics committees in Basel (EKBB, reference no. 377/09) and Côte d'Ivoire (reference no. 1993 MSHP/ CNER). The local authorities (i.e. village chiefs, school directors, teachers and medical staff) were informed about the objectives and procedures of the study. Literate parents and legal guardians of eligible schoolchildren were given an information sheet, whereas those who were illiterate were informed in lay terms by the teachers. Written informed consent was obtained from parents/guardians. Children assented orally. Participation was voluntary, and hence, children could withdraw at any time without further obligations.

At the end of the study, all participating schools were offered free treatment regardless of the infection status of the children. Praziquantel, manufactured by Bayer (single $40 \mathrm{mg} / \mathrm{kg}$ oral dose using a dose-pole) and albendazole, obtained from GlaxoSmithKline (single $400 \mathrm{mg}$ oral dose), were administered by medical staff.

\section{Selection of study settings}

The study was conducted within the frame of a multicountry project funded by the Schistosomiasis Consortium for Operational Research and Evaluation (SCORE) to assess the accuracy of a rapid diagnostic urine circulating cathodic antigen (CCA) cassette test for S. mansoni infections in different endemicity settings [9]. For the current investigation in Côte d'Ivoire, three $S$. mansoni endemicity settings were to be identified, as follows: (i) low S. mansoni endemicity setting (i.e. prevalence in school-aged children of 10-24\%); (ii) moderate $S$. mansoni endemicity setting (i.e. prevalence in school-aged children of 25-49\%); and (iii) setting where S. mansoni and S. haematobium are co-endemic. Recent research conducted in the district of Azaguié revealed a variety of schistosome endemicity scenarios [10-12], and hence this district, located approximately $40 \mathrm{~km}$ north of Abidjan (Figure 1), was chosen for the current investigation. In September 2010, we carried out a rapid screening in 11 schools. Twenty-five children, aged 8-12 years, were randomly selected by drawing lots in each school. Children provided one stool and one urine sample that were subjected to quality-controlled techniques.

Based on the screening results, three schools that fitted in the prevalence scheme indicated above were selected. All children aged 8-12 years were considered eligible for participation. According to sample size calculations for the overarching SCORE project, around 200 children from existing school lists were randomly selected in each of the three settings and invited to participate in the main study.

\section{Field procedures}

For the main study, in the early morning, right after school lessons had started, children were given plastic containers labelled with unique identification numbers (IDs) and invited to return the containers filled with a fresh morning stool sample (10-50 g) the following day. Upon sample collection, new empty containers were handed out for stool collection the next morning. This procedure was repeated over three days. Catch-up collections were done for two more days, and by then most of the children had submitted three stool samples. Additionally, after the children had provided stool samples, they were given another container and asked to provide a urine sample to be produced between 10:00 and 12:00 hours.

\section{Laboratory procedures}

Stool and urine collections were completed around noon. The samples were transferred to a parasitological laboratory at the Université de Cocody in Abidjan and processed the same day as follows. First, triplicate $41.7 \mathrm{mg}$ Kato-Katz thick smears were prepared from each stool sample [13]. The slides were allowed to clear for at least $30 \mathrm{~min}$ before examination under a microscope by one of five experienced laboratory technicians. The number of helminth eggs were counted and recorded for each species separately. Second, from each 


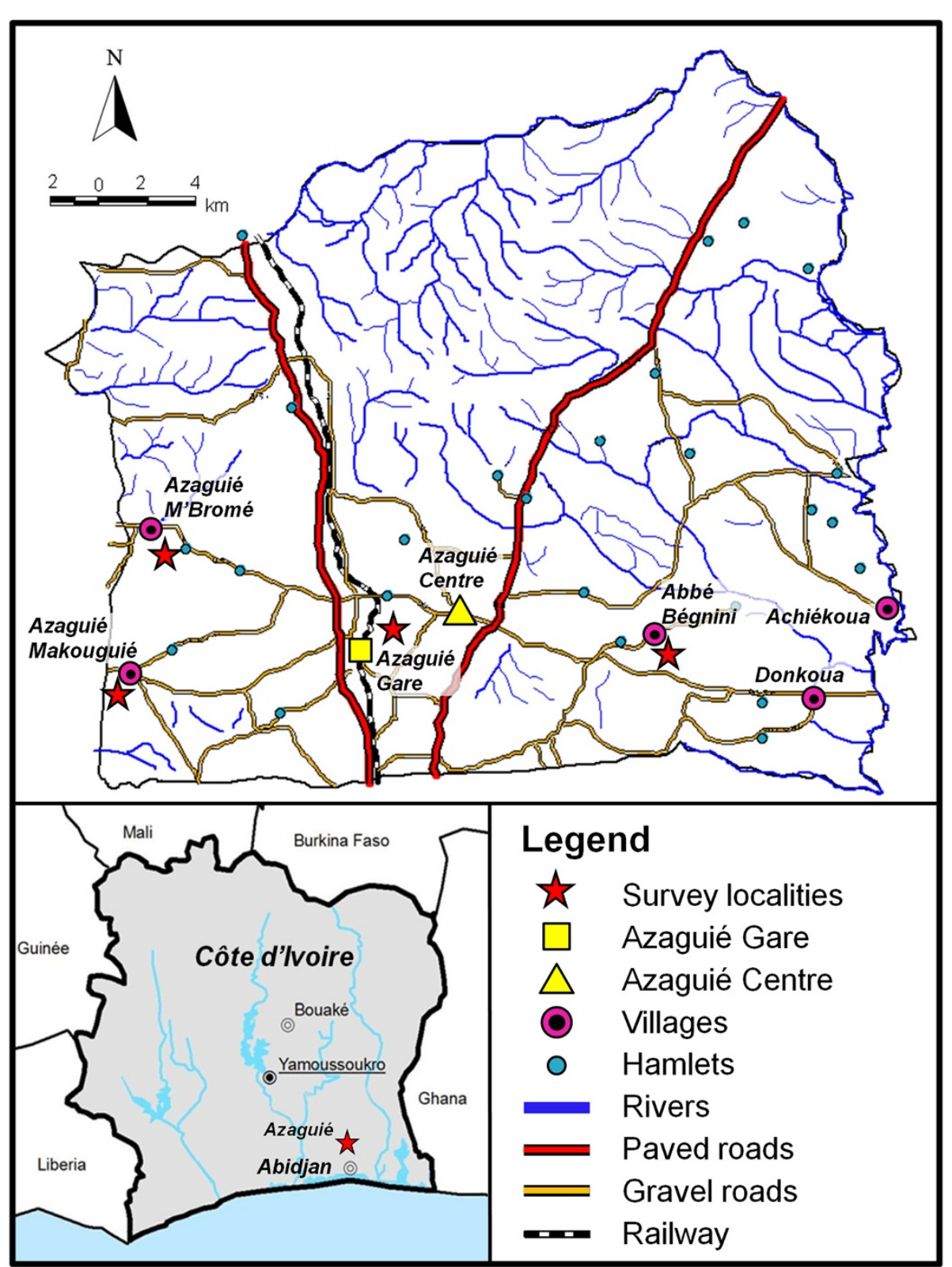

Figure 1 Map showing the district of Azaguié in south Côte d'Ivoire. Indicated are Azaguié town and its surrounding villages. Among the 11 schools included in the pre-screening, five were located in the surrounding villages and six were located in the Azaguie town area. The three settings selected for the in-depth studies (i.e. Azaguié M'Bromé/Azaguié Makouguié, rural; Abbé-Bégnini, peri-urban; Azaguié Gare, urban) are emphasised with red asterisks.

participant's second day stool sample, $\sim 1 \mathrm{~g}$ of stool was placed into a Falcon tube containing $10 \mathrm{ml}$ of sodium acetate-acetic acid-formalin (SAF) solution, broken and homogenized with a wooden spatula and vigorously shaken. Third, urine samples were shaken and $10 \mathrm{ml}$ filtered using a plastic syringe and filter-holders with 13-mm diameter filter (mesh size $20 \mu \mathrm{m}$ ) (Sefar AG; Heiden, Switzerland). Filters were removed with forceps, placed on microscope slides, a drop of Lugol's solution added and examined under a microscope. The number of S. haematobium eggs was counted and recorded.

Within 6-10 weeks of stool collection, the SAF-fixed samples were subjected to an ether-concentration method, using a standard protocol [14]. In brief, the SAF-fixed stool samples were re-suspended and filtered through a medical gauze placed in a plastic funnel into a centrifuge tube. The tube was centrifuged for $1 \mathrm{~min}$ at $500 \mathrm{~g}$. After centrifugation, the supernatant was discarded 
and $7 \mathrm{ml}$ of $0.85 \% \mathrm{NaCl}$ plus $2-3 \mathrm{ml}$ of ether were added to the remaining pellet. After shaking for $10-30 \mathrm{~s}$, the tube and its contents were centrifuged for $5 \mathrm{~min}$ at $500 \mathrm{~g}$. Finally, from the four layers formed, the three top layers were discarded. The bottom layer, including the sediment, was examined under a microscope. The number of helminth eggs were counted and recorded for each species separately. Intestinal protozoa were recorded semi-quantitatively as follow: (i) negative (no cysts or trophozoites in the entire sediment); (ii) light (one to five cysts or trophozoites per slide); (iii) moderate (one cyst or trophozoite per observation field at a magnification of $x 400$ or $x$ 500); and (iv) heavy (more than one cyst or trophozoite per observation field at a magnification of $\mathrm{x} 400$ or $\mathrm{x} 500$ ) [14].

\section{Statistical analysis}

Data were double entered and cross-checked using Epilnfo version 3.2 (Centers for Disease Control and Prevention; Atlanta, GA, USA). Statistical analyses were carried out using STATA version 10 (Stata Corporation; College Station, TX, USA).

Parasite species-specific data analysis was restricted to those children who had complete data records (i.e. three stool samples examined with triplicate Kato-Katz thick smears for S. mansoni and soil-transmitted helminths, one stool sample subjected to an ether-concentration method for helminths and intestinal protozoa, and three urine samples examined with a single urine filtration for S. haematobium).

For each individual, arithmetic mean egg counts of the helminths were calculated from the nine KatoKatz thick smears and three urine filtration readings. Helminth infection intensities were expressed as eggs per gram of stool (EPG) and eggs/10 ml of urine (for $S$. haematobium) and classified into light, moderate and heavy infection intensities, according to thresholds put forth by the World Health Organization (WHO) [1,15]. Helminth infection intensities were also estimated at the setting level, using the group's arithmetic mean faecal egg counts [16].

Dichotomous data are presented as proportion in the pre-screening as well as for the three main study settings. For the latter settings, multivariable logistic regression models were fitted for each parasite. The prevalence of a respective parasite was used as dependent variable (binary variable: present/absent) and the prevalence of each other parasite, sex (binary variable: male/female), age (continuous variable) and the study settings (categorical variable) were used as explanatory variables. As preparatory analysis for assessment of our multivariable logistic regressions, in each setting, univariable logistic regression was used to assess the association between each dependent variable (parasite infection) and each covariate (i.e. sex, age, village and other parasites). Subsequently, interactions between sex, age and village were assessed for each dependent variable. Next, village effects were assessed by considering a clustered structure in the models (xtlogit command in STATA). That was, we considered a random intercept for each village in the logistic regression model. Village effects for the respective parasites were fitted in each final model. At the end, a backward stepwise elimination procedure was applied, including interactions and village effect to determine significant association between parasitic infections and between parasitic infections and study setting. The explanatory variable with the highest $\mathrm{p}$-value in the multivariable logistic regression model was eliminated before re-running the multivariable logistic regression model and these iterations of elimination were continued as long as the values of the Akaike information criterion (AIC) of the new models were decreasing. A similar approach has already been successfully used in other studies [17]. A p-value below 0.05 was considered as statistically significant.

\section{Results}

\section{Pre-screening}

Table 1 shows the results of the initial screening carried out in 11 schools in the Azaguié district, south Côte d'Ivoire. According to triplicate Kato-Katz thick smears

Table 1 Prevalence of S. mansoni and S. haematobium, as assessed in an initial screening carried out in 11 schools in Azaguié district, south Côte d'Ivoire in September 2010

\begin{tabular}{lccl}
\hline School & \multicolumn{2}{l}{ No. (\%) of infected children } & Endemicity $^{\mathbf{a}}$ \\
\cline { 2 - 3 } & S. mansoni & S. haematobium & \\
\hline Abbé-Bégnini & $4(16)$ & 0 & Low \\
Achiékoua & 0 & 0 & Not selected \\
Ahoua 1 & $14(56)$ & $1(4)$ & Not selected \\
Ahoua 2 & $14(56)$ & $1(4)$ & Not selected \\
Ahoua 3 & $15(60)$ & $2(8)$ & Not selected \\
Azaguié Gare 1A & $9(36)$ & 0 & Moderate \\
Azaguié Gare 2A & $7(28)$ & 0 & Moderate \\
Azaguié Gare 2B & $9(36)$ & $3(12)$ & Moderate \\
Bambou & $11(44)$ & $1(4)$ & Not selected \\
Azaguié M'Bromé & $17(68)$ & $14(56)$ & Mixed \\
Azaguié Makouguié & $22(88)$ & $13(52)$ & Mixed
\end{tabular}

${ }^{a}$ Endemicity was set according to SCORE guidelines: prevalence of S. mansoni between $10 \%$ and $24 \%$ indicates low endemicity, prevalence of S. mansoni between $25 \%$ and $49 \%$ was considered moderate endemicity, co-existence of S. mansoni and S. haematobium indicates mixed endemicity.

In each school, the prevalence was assessed among 25 randomly selected children, aged 8-12 years. One stool sample was examined with triplicate Kato-Katz thick smears to determine the prevalence of S. mansoni, whereas one urine sample was subjected to a single filtration to assess the prevalence of S. haematobium. 
derived from a single stool sample obtained from 25 children per school, the prevalence of $S$. mansoni ranged between nil and $88 \%$. Abbé-Bégnini with a $S$. mansoni prevalence of $16 \%$ was the only low endemicity school, whereas the three schools in Azaguié Gare were determined as moderately endemic ( $S$. mansoni prevalences ranging between $25 \%$ and $49 \%$ ). S. haematobium infections, determined by single urine filtrations, were found in seven of the 11 schools. In five of these schools, only one to three children were infected. However, in Azaguié M'Bromé and Azaguié Makouguié, more than half of the children were infected with $S$. haematobium. Hence, the settings Abbé-Bégnini, Azaguié Gare and Azaguié M’Bromé/Azaguié Makouguié were selected, as they fitted SCORE's predefined classifications of low, moderate and mixed endemicity, respectively.

\section{Characteristics of study settings and population}

Adhering to SCORE guidelines, Abbé-Begnini (low endemicity $S$. mansoni setting), Azaguié Gare (moderate endemicity S. mansoni setting) and Azaguié M'Bromé/ Azaguié Makouguié (mixed S. mansoni-S. haematobium setting) were selected for an in-depth appraisal of schoolaged children's helminth and intestinal protozoan infection profiles. Interestingly, Azaguié M'Bromé/Azaguié Makouguié, Abbé-Bégnini and Azaguié Gare (Figure 1) were located in rural, peri-urban and urban areas, respectively. The proportions of children with complete data records from the sampling of stool and urine were 77.3\% in Azaguié M’Bromé/Azaguié Makouguié, 62.4\% in Abbé-Bégnini and 59.1\% in Azaguié Gare.

Azaguié M’Bromé and Azaguié Makouguié, two small villages located approximately 9 and $12 \mathrm{~km}$ west of Azaguié town, are typical rural settings. These two villages are difficult to access via gravel roads that are infrequently used by private transport. People are mainly engaged in subsistence farming. There is no tap water supply and households lack sanitation facilities, and hence the population practices open defecation. In each village, there is a primary school. Additionally, in Azaguié M’Bromé, there is a primary health care centre.

Abbé-Bégnini is a peri-urban village located approximately $5 \mathrm{~km}$ east of the centre part of Azaguié town. The village is reachable on a tarmac road by private and public transport. Subsistence farming is the main source of income. Sanitation coverage is low and inhabitants depend on open surface water from the nearby river for household use and on two water pumps for drinking and cooking. There is one primary health care centre.

Azaguié Gare is an urban neighbourhood of Azaguié town, which has excellent transport connections to Abidjan (bus, car and train). The population mainly consists of civil servants, artisans, traders and some farmers. Most of the inhabitants have access to tap water and only few use public man-made wells. Latrines with cemented slabs or flush toilets are common. The town has several private primary health care centres and a public secondary health facility.

\section{Intestinal parasitic infections}

Figure 2 shows the study adherence. Overall, 170, 146 and 130 schoolchildren had complete parasitological data in Azaguié M’Bromé/Azaguié Makouguié, AbbéBégnini and Azaguié Gare, respectively. Table 2 shows the infection prevalence of intestinal parasites in the main study, stratified by setting. In rural Azaguié M’Bromé/Azaguié Makouguié, very high prevalences of both S. mansoni and S. haematobium were found (91.8\% and $65.3 \%$, respectively). T. trichiura was the predominant soil-transmitted helminth (56.5\%). While hookworm was also common (44.7\%), the prevalence of A. lumbricoides was considerably lower (11.2\%). E. coli with a prevalence of $31.8 \%$ was found to be the predominant intestinal protozoon infection, followed by Endolimax nana (28.8\%), Blastocystis hominis (15.9\%) and G. intestinalis (8.8\%).

In peri-urban Abbé-Bégnini, hookworm and S. mansoni showed the highest prevalences, $41.1 \%$ and $32.9 \%$, respectively. The prevalence of $S$. haematobium, $T$. trichiura and A. lumbricoides were below $10 \%$. E. coli and E. nana were the predominant intestinal protozoa with respective prevalences of $26.7 \%$ and $24.7 \%$.

In urban Azaguié Gare, more than half (53.1\%) of the children harboured S. mansoni. The prevalences of hookworm, T. trichiura and A. lumbricoides were 20.8\%, $15.4 \%$ and $13.1 \%$, respectively. Only one child $(0.8 \%)$ was found with $S$. haematobium eggs in the urine. In this setting, $E$. nana was the predominant intestinal protozoon species (42.3\%), followed by E. coli $(25.4 \%)$ and B. hominis (10.2\%).

\section{Infection intensities}

Infection intensities, expressed as group arithmetic mean faecal egg counts, showed some heterogeneity between settings (Table 3). Infection intensity classes for helminths and intestinal protozoa, stratified by setting, are summarised in Table 4. In rural Azaguié M’Bromé/Azaguié Makouguié, more than two-thirds of S. mansoni-infected children had either a moderate (35.2) or a heavy (41.7\%) infection. S. haematobium, hookworm, T. trichiura and $A$. lumbricoides infections were mainly of light intensities. Most of the children were heavily infected with intestinal protozoa. In peri-urban Abbé-Bégnini, $97.0 \%$ of the infected schoolchildren showed light helminth infection intensities. Intestinal protozoan infection intensities showed no clear intensity patterns. In urban Azaguié Gare, all helminth infections were of light intensities. 


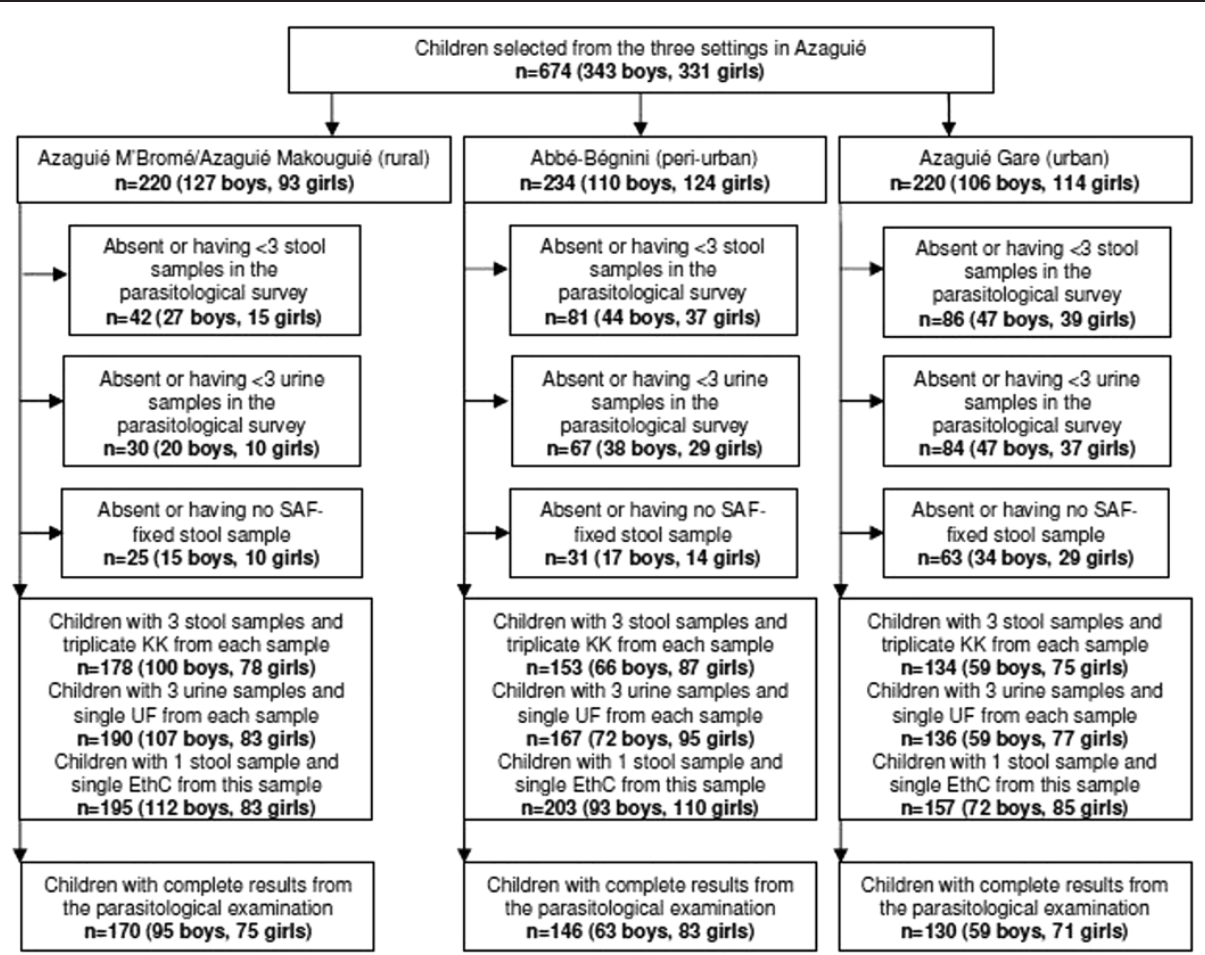

Figure 2 Flow chart detailing the study participation and adherence. Children were selected from three different settings in Azaguié district, south Côte d'Ivoire in October and November 2010. KK, Kato-Katz technique; UF, urine filtration method; EthC, ether-concentration method.

Intestinal protozoan infections were light (58.4\%) or moderate $(36.5 \%)$ and only 13 children showed a heavy infection.

\section{Multiparasitism}

Figure 3 shows the patterns of multiple species intestinal parasite infections, stratified by setting. In the rural setting, the prevalence of schoolchildren with single, dual or multiple species helminth infection was 9.2\%, $22.4 \%$ and $61.6 \%$, respectively. In the peri-urban setting, $42.1 \%$, $22.1 \%$ and $6.2 \%$ of the children harboured one, two or at least three helminth species concurrently. In the urban setting, single helminth species infections were most common $(41.2 \%)$, whereas dual species helminth infections were found in $18.2 \%$ of the children investigated (Figure 3A).

Multiple species intestinal protozoan infections were assessed with a single ether-concentration test. In rural Azaguié M'Bromé/Azaguié Makouguié, 29.9\% of the children harboured single species intestinal protozoan infections, whereas $21.3 \%$ harboured two species of intestinal protozoa concurrently. An infection with three or more intestinal protozoan species was found in $12.5 \%$ of the children. In peri-urban Abbé-Bégnini, 36.5\%, $29.3 \%$ and $5.1 \%$ showed single, dual or multiple species intestinal protozoan infections. In urban Azaguié Gare, $40.1 \%$ harboured single species intestinal protozoan infections, whereas dual or multiple species infections were found in $21.6 \%$ and $5.4 \%$, respectively (Figure 3B).

Considering all intestinal parasite species (i.e. helminths and intestinal protozoa), the prevalence of single, dual and multiple species infections in the rural setting was $2.9 \%, 15.2 \%$ and $79.5 \%$, respectively. Two schoolchildren harboured eight or 10 intestinal parasites concurrently. In the peri-urban setting, 39.4\%, 32.4\% and $28.2 \%$ of the schoolchildren harboured single, dual and multiple species infections, respectively. In the urban setting, the prevalence of single, dual and multiple intestinal parasite infections was $23.0 \%, 35.7 \%$ and $33.3 \%$, respectively (Figure 3C).

\section{Parasite associations}

All significant associations $(\mathrm{p}<0.05)$ between intestinal parasites, sex, age and setting resulting from the multivariable logistic regression models are summarised in Table 5. S. mansoni infection showed significant positive associations with $S$. haematobium (odds ratio $(\mathrm{OR})=$ 4.81, 95\% confidence interval $(\mathrm{CI})=1.79-12.93)$ and T. trichiura $(\mathrm{OR}=2.74,95 \% \mathrm{CI}=1.34-5.60)$. The odds of being infected with $S$. mansoni increased with age $(\mathrm{OR}=1.55,95 \% \mathrm{CI}=1.29-1.87)$. A. lumbricoides was significantly associated with $T$. trichiura $(\mathrm{OR}=4.24,95 \%$ $\mathrm{CI}=1.96-9.19)$ and hookworm $(\mathrm{OR}=2.34,95 \% \mathrm{CI}=$ 1.14-4.41). Boys were more likely to be infected with 
Table 2 Prevalence of helminths and intestinal protozoa infections in three settings of Azaguié district, south Côte d'Ivoire in October and November 2010

\begin{tabular}{|c|c|c|c|c|c|c|}
\hline \multirow[t]{3}{*}{ Intestinal parasite } & \multicolumn{6}{|l|}{ Study setting $^{a}$} \\
\hline & \multicolumn{2}{|c|}{ Azaguié M’Bromé/Azaguié Makouguié (rural) } & \multicolumn{2}{|c|}{ Abbé-Begnini (peri-urban) } & \multicolumn{2}{|c|}{ Azaguié Gare (urban) } \\
\hline & No. of infected & $\%(95 \% \mathrm{Cl})$ & No. of infected & $\%(95 \% \mathrm{Cl})$ & No. of infected & $\%(95 \% \mathrm{Cl})$ \\
\hline \multicolumn{7}{|l|}{ Helminths } \\
\hline Schistosoma mansoni & 156 & $91.8(87.6-95.9)$ & 48 & $32.9(25.2-40.6)$ & 69 & $53.1(44.4-61.8)$ \\
\hline Schistosoma haematobium & 111 & $65.3(58.1-72.5)$ & 6 & $4.1(0.1-7.4)$ & 1 & $0.8(0-2.0)$ \\
\hline Trichuris trichiura & 96 & $56.5(48.9-63.9)$ & 10 & $6.9(2.7-10.9)$ & 20 & $15.4(9.1-21.7)$ \\
\hline Hookworm & 76 & $44.7(37.2-52.3)$ & 60 & $41.1(33.0-49.2)$ & 27 & $20.8(13.7-27.8)$ \\
\hline Ascaris lumbricoides & 19 & $11.2(6.4-15.9)$ & 10 & $6.9(2.7-10.9)$ & 17 & $13.1(7.2-18.9)$ \\
\hline \multicolumn{7}{|l|}{ Intestinal protozoa } \\
\hline Entamoeba coli & 54 & $31.8(24.7-38.8)$ & 39 & $26.7(19.5-33.9)$ & 33 & $25.4(17.8-32.9)$ \\
\hline Endolimax nana & 49 & $28.8(21.9-35.7)$ & 36 & $24.7(17.6-31.7)$ & 55 & $42.3(33.7-50.9)$ \\
\hline Blastocystis hominis & 27 & $15.9(10.7-22.3)$ & 14 & $9.6(4.8-14.4)$ & 14 & $10.8(5.4-16.2)$ \\
\hline Giardia intestinalis & 15 & $8.8(4.5-13.1)$ & 11 & $7.5(3.2-11.9)$ & 12 & $9.2(4.2-12.3)$ \\
\hline Chilomastix mesnili & 14 & $8.2(4.1-12.4)$ & 7 & $4.8(1.3-8.3)$ & 6 & $4.6(0.9-8.3)$ \\
\hline Entamoeba histolytica/E. dispar & 14 & $8.2(4.1-12.4)$ & 12 & $8.2(3.7-12.7)$ & 7 & $5.4(1.5-9.3)$ \\
\hline lodamoeba bütschlii & 9 & $5.3(1.9-8.7)$ & 8 & $5.5(1.7-9.2)$ & 10 & $7.7(3.1-12.3)$ \\
\hline Entamoeba hartmanni & 5 & $2.9(0.4-5.5)$ & 8 & $5.5(1.7-9.2)$ & 9 & $6.9(2.5-11.3)$ \\
\hline
\end{tabular}

${ }^{a} \mathrm{n}=170$ (rural setting), $\mathrm{n}=146$ (peri-urban setting), $\mathrm{n}=130$ (urban setting).

Diagnosis of S. mansoni and soil-transmitted helminths were based on nine Kato-Katz thick smears (three stool samples, each subjected to triplicate Kato-Katz).

Diagnosis of S. haematobium was based on three urine samples, each subjected to a single filtration. Diagnosis of intestinal protozoa was based on a single stool sample fixed in SAF that was examined with an ether-concentration technique.

$\mathrm{Cl}$, confidence interval.

hookworm than girls $(\mathrm{OR}=1.87,95 \% \mathrm{CI}=1.24-2.81)$. E. coli was significantly associated with $S$. haematobium $(\mathrm{OR}=1.73,95 \% \mathrm{CI}=1.09-2.72)$.

\section{Discussion}

We studied patterns of intestinal parasite infections (i.e. schistosomes, soil-transmitted helminths and intestinal protozoa) in school-aged children in three settings of south Côte d'Ivoire; a rural, peri-urban and urban area. An important aspect of parasitic disease control programmes is the ability to readily identify and reach people at highest risk of infection and associated morbidity. Often, the poorest people are the least accessible ones living in remote rural areas, and hence they are at highest risk of parasitic infection and other conditions of ill-health [18-20].

In this study, the assessment of children's prevalence and intensity of S. mansoni and soil-transmitted helminth infections was based on nine Kato-Katz thick smears derived from three stool samples. The day-to-day and intra-specimen variation in helminth egg output that compromise the sensitivity of the Kato-Katz technique, especially in areas of low infection intensity, is overcome by such a rigorous diagnostic approach [2123]. However, multiple stool sampling to increase the sensitivity of the Kato-Katz technique for helminth diagnosis might result in reduced compliance. Indeed, particularly in the urban setting, compliance for providing

Table 3 Arithmetic mean helminth eggs per gram of stool (EPG), stratified by setting

\begin{tabular}{|c|c|c|c|c|c|c|}
\hline & \multicolumn{2}{|c|}{ Azaguié M’Bromé/Azaguié Makouguié (rural) } & \multicolumn{2}{|c|}{ Abbé-Bégnini (peri-urban) } & \multicolumn{2}{|c|}{ Azaguié Gare (urban) } \\
\hline & Arithmetic mean & $95 \% \mathrm{Cl}$ & Arithmetic mean & $95 \% \mathrm{Cl}$ & Arithmetic mean & $95 \% \mathrm{Cl}$ \\
\hline Schistosoma mansoni & 482.7 & $388.1-577.4$ & 17.4 & $0.0-38.9$ & 62.4 & $36.2-88.5$ \\
\hline Schistosoma haematobium ${ }^{a}$ & 19.4 & $13.3-25.5$ & 2.3 & $0.4-4.2$ & 1.5 & $0.5-2.6$ \\
\hline Ascaris lumbricoides & 10.3 & $0.2-20.4$ & 46.3 & $5.5-87.2$ & 175.4 & $0.0-474.1$ \\
\hline Trichuris trichiura & 136.4 & $81.7-191.1$ & 9.4 & $0.0-23.2$ & 5.9 & $3.1-8.8$ \\
\hline Hookworm & 49.1 & $17.9-80.2$ & 56.7 & $23.8-89.6$ & 6.6 & $3.3-9.8$ \\
\hline
\end{tabular}

${ }^{\mathrm{a}}$ infection intensity expressed as eggs per $10 \mathrm{ml}$ of urine.

$\mathrm{Cl}$, confidence interval. 
Table 4 Categories of helminths and intestinal protozoan infection intensities, stratified by setting. Helminth infection intensities were categorized according to the classification of WHO $[1,15]$ and intestinal protozoan infection intensities were classified as described elsewhere [14]

\begin{tabular}{|c|c|c|c|c|c|c|c|c|c|}
\hline \multirow[t]{3}{*}{ Parasite } & \multicolumn{9}{|c|}{ Study setting } \\
\hline & \multicolumn{3}{|c|}{ Azaguié M’Bromé/Azaguié Makouguié (rural) } & \multicolumn{3}{|c|}{ Abbé-Begnini (peri-urban) } & \multicolumn{3}{|c|}{ Azaguié Gare (urban) } \\
\hline & $\begin{array}{l}\text { Light } \\
\text { (\%) }\end{array}$ & $\begin{array}{l}\text { Moderate } \\
(\%)\end{array}$ & $\begin{array}{l}\text { Heavy } \\
(\%)\end{array}$ & $\begin{array}{l}\text { Light } \\
(\%)\end{array}$ & $\begin{array}{l}\text { Moderate } \\
(\%)\end{array}$ & $\begin{array}{l}\text { Heavy } \\
\text { (\%) }\end{array}$ & $\begin{array}{l}\text { Light } \\
(\%)\end{array}$ & $\begin{array}{l}\text { Moderate } \\
(\%)\end{array}$ & $\begin{array}{l}\text { Heavy } \\
\text { (\%) }\end{array}$ \\
\hline \multicolumn{10}{|l|}{ Helminths } \\
\hline Schistosoma mansoni & $36(23.1)$ & $55(35.2)$ & $65(41.7)$ & $46(95.8)$ & $1(2.1)$ & $1(2.1)$ & $47(68.1)$ & $17(24.6)$ & $5(7.2)$ \\
\hline Schistosoma haematobium ${ }^{a}$ & $91(82.0)$ & - & $20(18.0)$ & $5(83.3)$ & - & $1(16.7)$ & 0 & - & $1(100)$ \\
\hline Hookworm & $75(100)$ & 0 & 0 & $59(98.3)$ & $1(1.7)$ & 0 & $27(100)$ & 0 & 0 \\
\hline Trichuris trichiura & $95(95.0)$ & $5(5.0)$ & 0 & $10(90.9)$ & $1(9.1)$ & 0 & $20(100)$ & 0 & 0 \\
\hline Ascaris lumbricoides & $19(100)$ & 0 & 0 & $10(100)$ & 0 & 0 & $16(94.1)$ & $1(5.9)$ & 0 \\
\hline \multicolumn{10}{|l|}{ Intestinal protozoa } \\
\hline Entamoeba coli & $11(20.4)$ & $18(33.3)$ & $25(46.3)$ & $7(17.9)$ & $14(35.9)$ & $18(46.2)$ & $15(50.0)$ & $12(40.0)$ & $3(10.0)$ \\
\hline Endolimax nana & 14 (28.6) & $9(18.4)$ & $26(53.1)$ & $7(19.4)$ & $13(36.1)$ & $17(47.2)$ & $26(46.4)$ & $23(41.1)$ & $7(12.5)$ \\
\hline Blastocystis hominis & $8(33.3)$ & $5(20.9)$ & $11(45.8)$ & $7(50.0)$ & $3(21.4)$ & $1(7.1)$ & $9(64.3)$ & $5(35.7)$ & 0 \\
\hline Giardia intestinalis & $1(6.7)$ & $4(26.7)$ & $10(66.7)$ & $1(9.1)$ & $3(27.3)$ & $7(63.6)$ & $8(66.7)$ & $3(25.0)$ & $1(8.3)$ \\
\hline Chilomastix mesnili & $2(14.3)$ & $4(28.6)$ & $8(57.1)$ & $1(14.3)$ & $1(14.3)$ & $5(71.4)$ & $3(50.0)$ & $2(33.3)$ & $1(16.7)$ \\
\hline Entamoeba histolytica/E. dispar & $4(28.6)$ & $8(57.1)$ & $2(14.3)$ & $3(25.0)$ & $4(33.3)$ & $5(41.7)$ & $6(66.7)$ & $2(22.2)$ & $1(11.1)$ \\
\hline lodamoeba bütschlii & 0 & $3(33.3)$ & $6(66.7)$ & $4(50.0)$ & $1(12.5)$ & $3(33.3)$ & $8(80.0)$ & $2(20.0)$ & 0 \\
\hline Entamoeba hartmanni & $1(20.0)$ & $1(20.0)$ & $3(60.0)$ & $4(50.0)$ & $2(25.0)$ & $2(25.0)$ & $8(88.9)$ & $1(11.1)$ & 0 \\
\hline
\end{tabular}

${ }^{a}$ Infection intensity cut-off for S. haematobium are $1-49$ eggs per $10 \mathrm{ml}$ of urine (light) and $\geq 50$ eggs per $10 \mathrm{ml}$ of urine (heavy).

The study was carried out in Azaguié, south Côte d'Ivoire in October and November 2010. S. mansoni and soil-transmitted helminth infection intensities were based on the reading of nine Kato-Katz thick smears. Intestinal protozoan infection intensities were based on one SAF-fixed stool sample subjected to an etherconcentration technique. Three urine filtrations were done to determine infection intensities of S. haematobium.

all three stool samples was considerably lower than in the rural and peri-urban settings and this might have introduced some bias.

Another aspect of our study worth highlighting is the following. After our pre-screening based on a single stool sample, and then employing a more rigorous diagnostic approach, the previously anticipated $S$. mansoni endemicity levels were considerably overrun. As predicted by mathematical modelling [24] and confirmed in field studies, repeated stool sampling with multiple Kato-Katz thick smears prepared from individual stool samples, result in considerable increases of the observed prevalence of $S$. mansoni $[21,23,25]$. The same observations have also been made for soil-transmitted helminths [22,26]. It follows that if rapid screenings are performed to reliably select a study setting or treatment scheme according to prevalence estimates, more sensitive diagnostic tools as alternatives to a rigorous sampling approach (i.e. collecting multiple stool samples and examining them with multiple Kato-Katz thick smears or other methods) are needed, particularly in areas with low transmission rates.

The current study confirms that schoolchildren from a rural setting are at higher risk of helminth infections than their counterparts living in peri-urban or urban settings. The remoteness of the rural setting, characterized by the absence of key infrastructures (e.g. tarmac road, health facilities, tap water and basic sanitation) play important roles. Our observations are in line with previous epidemiological surveys. Indeed, unsafe hygiene, water and sanitation and inadequate management of the environment exacerbate parasite infections in general, and helminth infections in particular [7,19,20,27,28]. Greatest differences between the prevalence of helminth infections, as a function of the study setting, were found with regard to the two schistosome species. Besides socioeconomic risk factors, the transmission of $S$. mansoni and $S$. haematobium is governed by intermediate host snails $[6,8,29]$, and hence the availability of suitable snail habitats seem to vary considerably between the three settings even at this small-scale. While it is commonly believed that schistosomiasis is a "rural disease", some studies have shown high prevalence in urban settings [30]. Hence, detailed malacological investigations are needed to deepen our understanding of the epidemiology of schistosomiasis with regard to the level of urbanization. Interestingly, similar hookworm prevalences were found in the rural and peri-urban settings. This observation could be explained by the fact that the behaviour of the schoolchildren with regard to hygiene and faecal management in particular is similar. Since open defecation is widely practiced in these communities, efforts must be made to improve 

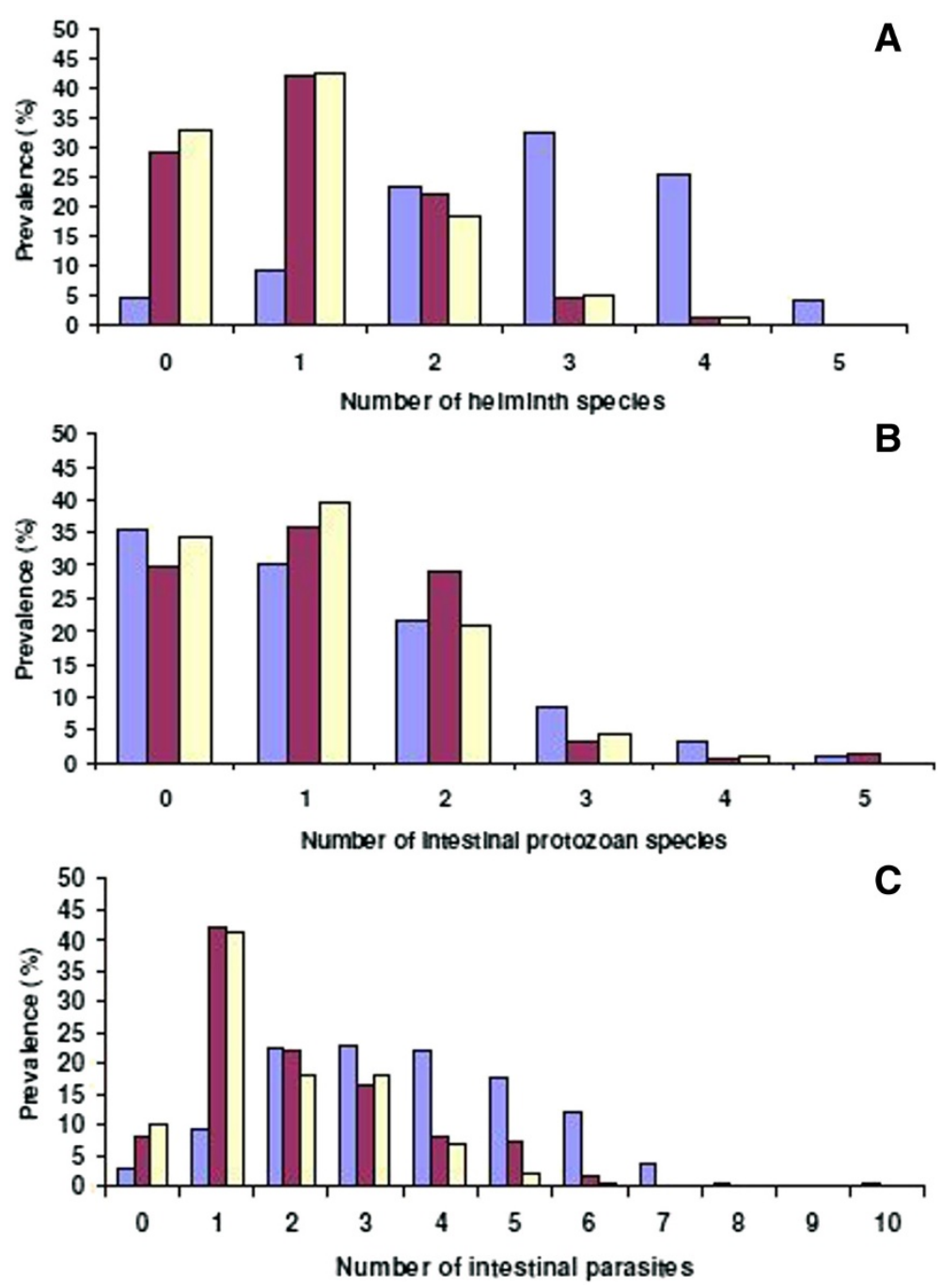

Figure 3 Multiple intestinal parasitic infections among schoolchildren aged 8-12 years in three settings of Azaguié district, south Côte d'Ivoire, in October and November 2010. Blue bars indicate rural Azaguié M'Bromé/Azaguié Makouguié; purple bars indicate peri-urban AbbéBégnini; and yellow bars indicate urban Azaguié Gare. (A) Number of helminth species diagnosed per child; (B) number of intestinal protozoa species diagnosed per child; (C) number of intestinal parasites (helminths and intestinal protozoa) diagnosed per child.

sanitation, which in turn will have major ramification on other neglected tropical diseases such as amoebiasis and giardiasis [28,31].

Interestingly, the prevalence of intestinal protozoan infections among schoolchildren was found to be similar in the three settings. This might indicate that hygiene related to food consumption among schoolchildren is similar and needs to be improved. The two predominant intestinal protozoan species in the three settings under investigation are E. coli and E. nana. This observation is in agreement with previous studies done in different parts of Côte d'Ivoire [10,31,32].

Regarding intestinal parasite infection intensities, with the exception of $S$. mansoni in the rural setting, all other helminth infections were of light intensities whatever the setting. The observed moderate and heavy S. mansoni infections in the rural setting might be explained by high transmission in the absence of preventive and curative measures. Indeed, we are not aware of large-scale prior deworming activities in the district of Azaguié. There was a tendency of infection intensities of intestinal protozoa to decrease from rural to urban settings. Socioeconomic factors and educational attainment increase from rural to urban settings, and hence might explain the observed decrease in the intensities of intestinal protozoa.

Our study also confirms that multiparasitism is pervasive as observed elsewhere in sub-Saharan Africa and Asia [17,33-37]. We found two children in the rural setting harbouring eight or 10 different intestinal parasite species concurrently. This high number of parasite species in a 
Table 5 Significant associations of intestinal parasitic infections among schoolchildren in Azaguié district, south Côte d'Ivoire, in October and November 2010 ( $n=446)$

\begin{tabular}{lll}
\hline Parasite & Association & Adjusted OR (95\% C \\
\hline Schistosomiasis & & \\
Schistosoma mansoni & S. haematobium & $4.81(1.79-12.93)$ \\
& T. trichiura & $2.74(1.34-5.60)$ \\
& Age & $1.55(1.29-1.87)$ \\
Schistosoma haematobium & S. mansoni & $4.09(1.65-10.84)$ \\
Soil-transmitted helminths & & \\
Ascaris lumbricoides & T. trichiura & $4.24(1.96-9.19)$ \\
& Hookworm & $2.34(1.14-4.41)$ \\
Trichuris trichiura & Rural setting & $0.31(0.13-0.73)$ \\
Hookworm & S. mansoni & $2.89(1.42-5.91)$ \\
& A. lumbricoides & $4.14(1.90-8.99)$ \\
Intestinal protozoa & A. lumbricoides & $3.03(1.56-5.87)$ \\
Endolimax nana & Sex & $1.87(1.24-2.81)$ \\
& & \\
Entamoeba coli & B. hominis & $2.39(1.33-4.29)$ \\
Blastocystis hominis & Peri-urban setting & $0.44(0.26-0.74)$ \\
\hline Assocatons betwe & R. naral setting & $0.50(0.31-0.82)$ \\
& S. haematobium & $1.73(1.09-2.72)$ \\
& E. nana & $2.44(1.37-4.35)$ \\
\hline
\end{tabular}

Associations between a particular parasite (as binary variable; reference, absence) as dependent variable and age (as continuous variable), sex (as binary variable; reference, female), study setting (as categorical variable; reference, urban setting) and any of the remaining parasites (as binary variable; reference, absence) were analysed with multivariable logistic regression models, performing a stepwise backward elimination procedure. $\mathrm{Cl}$, confidence interval; OR, odds ratio.

single host is an alarming situation, as multiple species infection may increase susceptibility to other parasites [38-40]. Associations between different parasite species, as well as the influence of age, sex and study setting, have been assessed in the current investigation. Of particular note is the strong positive association between $S$. mansoni and S. haematobium with an adjusted OR above four. Moreover, we found a significant association between S. haematobium and E. coli, which has not been described in the literature before. Boys and girls were at the same level of exposure to helminths and intestinal protozoa, except for hookworm, as boys showed significantly higher infection prevalence than girls. Interestingly, a previous study carried out by Keiser et al. (2002) in western Côte d'Ivoire found that hookworm infections were significantly more often diagnosed in girls $[17,35]$. Behavioural and socioeconomic factors might explain this observed difference. Our study is in line with previous investigations that the risk of becoming infected with S. mansoni increases with age in children $[41,42]$. To date, the effects of parasite interactions on the human body remain poorly understood. Without a deeper understanding of such parasite interactions, the effectiveness of parasitic disease control programmes are compromised.

\section{Conclusion}

Continued and concerted efforts should be made by control programmes to reach rural school-aged children and other high-risk groups in the most remote areas, as they are the least accessed ones whose health and wellbeing is insufficiently accounted for by policy makers. Improvement of safe water supply and sanitation facilities by the construction of toilets could significantly reduce the burden of parasitic diseases in the rural and peri-urban settings studied here. In addition, particular importance might be given to health education at the district level. Control programmes should carefully consider the benefits of truly integrated (i.e. inter-programmatic and inter-sectoral) strategies. The findings of the present study may provide useful information for such integrated strategies to overcome the public health burden of intestinal parasitic infections in the Azaguié district in south Côte d'Ivoire in particular, and in other settings of the humid tropics.

\section{Competing interests}

The authors declare that they have no conflict of interest concerning the work reported in this paper.

\section{Acknowledgements}

We are grateful to the authorities of the Azaguié district, who received us warmly and greatly facilitated a smooth implementation of our study. We are indebted to Dr. Koutouan Y. N'Gbesso, head of the Azaguié district health centre for the strong implication in the field work, and Prof. Bassirou Bonfoh, Director General of the Centre Suisse de Recherches Scientifiques en Côte d'Ivoire, for his interest and continued support in all of our work. Many thanks are addressed to Mr. Laurent K. Lohourignon for expert help in the field and laboratory. We thank the primary school teachers who were involved in the study, as without their availability and help, the work reported here would not have been possible. Last but not least, we are grateful to the children for their enthusiastic participation.

\section{Author details}

1Department of Epidemiology and Public Health, Swiss Tropical and Public Health Institute, P.O. Box, CH-4002 Basel, Switzerland. 'University of Basel, P.O. Box, CH-4003 Basel, Switzerland. ${ }^{3}$ Unité de Formation et de Recherche Biosciences, Université de Cocody, 22 BP 770, Abidjan 22, Côte d'Ivoire. ${ }^{4}$ Centre Suisse de Recherches Scientifiques en Côte d'Ivoire, 01 BP 1303, Abidjan 01, Côte d'Ivoire.

\section{Authors' contributions}

JTC, TF, SK, JU and EKN designed the study; JTC, KDS, SK, MO and EKN implemented the study; JTC managed the data; JTC, TF, SK, DH, JU and EKN analysed and interpreted the data; JTC wrote the first draft of the paper; TF, SK, JU and EKN revised the paper. All authors read and approved the final version of the manuscript before submission.

Received: 17 February 2012 Accepted: 6 July 2012

Published: 6 July 2012

\section{References}

1. WHO: Prevention and control of schistosomiasis and soil-transmitted helminthiasis: first report of the joint WHO expert committees. WHO Tech Rep Ser 2002, 912:1-57.

2. Hotez PJ, Molyneux DH, Fenwick A, Ottesen E, Ehrlich Sachs S, Sachs JD: Incorporating a rapid-impact package for neglected tropical diseases 
with programs for HIV/AIDS, tuberculosis, and malaria. PLOS Med 2006, 3:e102.

3. Stanley SL Jr: Amoebiasis. Lancet 2003, 361:1025-1034.

4. Minenoa T, Avery MA: Giardiasis: recent progress in chemotherapy and drug development. Curr Pharm Des 2003, 9:841-855

5. Hotez PJ, Fenwick A, Savioli L, Molyneux DH: Rescuing the bottom billion through control of neglected tropical diseases. Lancet 2009, 373 : 1570-1575.

6. Yakubu N, Musa G, Yakubu SE: Seasonal changes in the distribution and infection rate of schistosome intermediate hosts in River Kubanni and its tributaries. Bio Res Com 2003, 15:207-214.

7. Wang LD, Guo JG, Wu XH, Chen HG, Wang TP, Zhu SP, Zhang ZH, Steinmann P, Yang GJ, Wang SP, et al: China's new strategy to block Schistosoma japonicum transmission: experiences and impact beyond schistosomiasis. Trop Med Int Health 2009, 14:1475-1483.

8. Aagaard-Hansen J, Mwanga JR, Bruun B: Social science perspectives on schistosomiasis control in Africa: past trends and future directions. Parasitology 2009, 136:1747-1758

9. Coulibaly JT, Knopp S, N'Guessan NA, Silué KD, Fürst T, Lohourignon LK, Brou JK, N'Gbesso YK, Vounatsou P, N'Goran EK, et al: Accuracy of urine circulating cathodic antigen (CCA) test for Schistosoma mansoni diagnosis in different settings of Côte d'Ivoire. PLoS Negl Trop Dis 2011, 5:e1384.

10. Ouattara M, N'Guessan NA, Yapi A, N'Goran EK: Prevalence and spatial distribution of Entamoeba histolytica/dispar and Giardia lamblia among schoolchildren in Agboville area (Côte d'Ivoire). PLoS Negl Trop Dis 2010, 4:e574.

11. N'Guessan NA, Acka CA, Utzinger J, N'Goran EK: Identification des regions à haute risque de schistosome en Côte d'Ivoire. Bull Soc Pathol Exot 2006 100:119-123. in French.

12. Glinz D, Silué KD, Knopp S, Lohourignon LK, Yao KP, Steinmann P, Rinaldi L, Cringoli G, N'Goran EK, Utzinger J: Comparing diagnostic accuracy of Kato-Katz, Koga agar plate, ether-concentration, and FLOTAC for Schistosoma mansoni and soil-transmitted helminths. PLoS Negl Trop Dis 2010, 4:e754.

13. Katz N, Chaves A, Pellegrino J: A simple device for quantitative stool thicksmear technique in schistosomiasis mansoni. Rev Inst Med Trop São Paulo 1972, 14:397-400.

14. Utzinger J, Botero-Kleiven S, Castelli F, Chiodini PL, Edwards H, Kohler N, Gulletta M, Lebbad M, Manser M, Matthys B, et al: Microscopic diagnosis of sodium acetate-acetic acid-formalin-fixed stool samples for helminths and intestinal protozoa: a comparison among European reference laboratories. Clin Microbiol Infect 2008, 16:267-273.

15. WHO: Guideline for the evaluation of soil-transmitted helminthiasis and schistosomiasis at the community level: a guide for managers of control programmes. Geneva: World Health Organization; 1998. WHO/CTD/SIP/98.1).

16. Vercruysse J, Behnke JM, Albonico M, Ame SM, Angebault C, Bethony JM, Engels D, Guillard B, Nguyen TV, Kang G, et al: Assessment of the anthelmintic efficacy of albendazole in school children in seven countries where soil-transmitted helminths are endemic. PLoS Negl Trop Dis 2011, 5:e948.

17. Raso G, Luginbühl A, Adjoua CA, Tian-Bi NT, Silue KD, Matthys B, Vounatsou $P$, Wang $Y$, Dumas ME, Holmes $E$, et al: Multiple parasite infections and their relationship to self-reported morbidity in a community of rural Côte d'Ivoire. Int J Epidemiol 2004, 33:1092-1102.

18. Asaolu SO, Ofoezie IE: The role of health education and sanitation in the control of helminth infections. Acta Trop 2003, 86:283-294.

19. Hotez P: Hookworm and poverty. Ann N Y Acad Sci 2008, 1136:38-44

20. Huang $Y X$, Manderson L: The social and economic context and determinants of schistosomiasis japonica. Acta Trop 2005, 96:223-231.

21. Utzinger J, Booth M, N'Goran EK, Müller I, Tanner M, Lengeler C: Relative contribution of day-to-day and intra-specimen variation in faecal egg counts of Schistosoma mansoni before and after treatment with praziquantel. Parasitology 2001, 122:537-544.

22. Knopp S, Mgeni AF, Khamis IS, Steinmann P, Stothard JR, Rollinson D, Marti H, Utzinger J: Diagnosis of soil-transmitted helminths in the era of preventive chemotherapy: effect of multiple stool sampling and use of different diagnostic techniques. PLoS Negl Trop Dis 2008, 2:e331.

23. Enk MJ, Lima AC, Drummond SC, Schall VT, Coelho PM: The effect of the number of stool samples on the observed prevalence and the infection intensity with Schistosoma mansoni among a population in an area of low transmission. Acta Trop 2008, 108:222-228.

24. de Vlas SJ, Gryseels B: Underestimation of Schistosoma mansoni prevalences. Parasitol Today 1992, 8:274-277.

25. Booth M, Vounatsou P, N'Goran EK, Tanner M, Utzinger J: The influence of sampling effort and the performance of the Kato-Katz technique in diagnosing Schistosoma mansoni and hookworm co-infections in rural Côte d'Ivoire. Parasitology 2003, 127:525-531.

26. Steinmann $P, D u Z W$, Wang LB, Wang XZ, Jiang JY, Li LH, Marti $H$, Zhou XN, Utzinger J: Extensive multiparasitism in a village of Yunnan province, People's Republic of China, revealed by a suite of diagnostic methods. Am J Trop Med Hyg 2008, 78:760-769.

27. King CH: Parasites and poverty: the case of schistosomiasis. Acta Trop 2010, 113:95-104.

28. Ziegelbauer K, Speich B, Mäusezahl D, Bos R, Keiser J, Utzinger J: Effect of sanitation on soil-transmitted helminth infection: systematic review and meta-analysis. PLoS Med 2012, 9:e1001162.

29. Stensgaard AS, Utzinger J, Vounatsou P, Hürlimann E, Schur N, Saarnak CFL, Simoonga C, Mubita P, Kabatereine NB, Tchuem Tchuente LA, 2012, et al: Large-scale determinants of intestinal schistosomiasis and intermediate host snail distribution across Africa: does climate matter? Acta Trop 2012. doi:10.1016/j.actatropica.2011.11.010. in press.

30. Matthys B, Tschannen AB, Tian-Bi NT, Comoé H, Diabaté S, Traoré M, Vounatsou P, Raso G, Gosoniu L, Tanner M, et al: Risk factors for Schistosoma mansoni and hookworm in urban farming communities in western Côte d'Ivoire. Trop Med Int Health 2007, 12:709-723.

31. Keiser J, N'Goran EK, Traoré M, Lohourignon KL, Singer BH, Lengeler C, Tanner M, Utzinger J: Polyparasitism with Schistosoma mansoni, geohelminths, and intestinal protozoa in rural Côte d'Ivoire. J Parasitol 2002, 88:461-466

32. Traoré SG, Odermatt $P$, Bonfoh B, Utzinger J, Aka ND, Adoubryn KD, Assoumou A, Dreyfuss G, Koussemon M: No Paragonimus in high-risk groups in Côte d'Ivoire, but considerable prevalence of helminths and intestinal protozoon infections. Parasit Vectors 2011, 4:96.

33. Brooker S, Miguel EA, Moulin S, Luoba Al, Bundy DAP, Kremer M Epidemiology of single and multiple species of helminth infections among school children in Busia district, Kenya. East Afr Med J 2000, 77:157-161.

34. Chunge RN, Karumba N, Ouma JH, Thiongo FW, Sturrock RF, Butterworth AE: Polyparasitism in two rural communities with endemic Schistosoma mansoni infection in Machakos district, Kenya. J Trop Med Hyg 1995, 98:440-444

35. Keiser J, N'Goran EK, Singer BH, Lengeler C, Tanner M, Utzinger J: Association between Schistosoma mansoni and hookworm infections among schoolchildren in Côte d'Ivoire. Acta Trop 2002, 84:31-41.

36. Pullan $\mathrm{R}$, Brooker $\mathrm{S}$ : The health impact of polyparasitism in humans: are we under-estimating the burden of parasitic diseases? Parasitology 2008 135:783-794.

37. Steinmann P, Utzinger J, Du ZW, Zhou XN: Multiparasitism: a neglected reality on global, regional and local scale. Adv Parasitol 2010, 73:21-50

38. Druilhe $P$, Tall A, Sokhna C: Worms can worsen malaria: towards a new means to roll back malaria? Trends Parasitol 2005, 21:359-362.

39. Mwangi TW, Bethony JM, Brooker S: Malaria and helminth interactions in humans: an epidemiological viewpoint. Ann Trop Med Parasitol 2006, 100:551-570.

40. Nacher M: Interactions between worm infections and malaria. Clin Rev Allergy Immunol 2004, 26:85-92.

41. Hotez PJ, Brindley PJ, Bethony JM, King CH, Pearce EJ, Jacobson J: Helminth infections: the great neglected tropical diseases. J Clin Invest 2008, 118:1311-1321.

42. Tchuem Tchuenté LT: Control of schistosomiasis and soil-transmitted helminthiasis in sub-Saharan Africa: challenges and prospects. In Current topics in tropical medicine. Edited by Rodriguez-Morales AJ. Croatia: InTech; 2012:359-376.

doi:10.1186/1756-3305-5-135

Cite this article as: Coulibaly et al:: Intestinal parasitic infections in schoolchildren in different settings of Côte d'Ivoire: effect of diagnostic approach and implications for control. Parasites \& Vectors 2012 5:135. 